\title{
The fungal ecology of seabird nesting sites in the Falkland Islands indicates a niche for mycoparasites
}

\author{
Jacob Hargreaves ${ }^{a, b}$, Paul Brickle ${ }^{b, c}$, Pieter van West ${ }^{a^{*}}$ \\ ${ }^{a}$ Aberdeen Oomycete Laboratory, College of Life Sciences and Medicine, Institute of Medical \\ Sciences, University of Aberdeen, Aberdeen, UK.

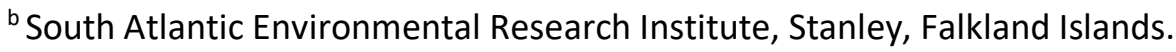

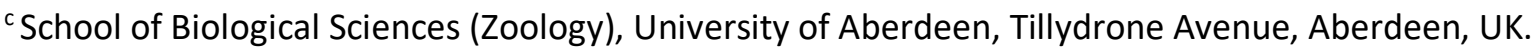

\section{Abstract}

Soil fungal communities are complex and heterogeneous, possessing important functions in most terrestrial ecological systems. Their study has been advanced due to the development of highthroughput sequencing, which allow for complex fungal communities to be described with techniques such as metabarcoding. The Falkland Islands contain large populations of breeding seabirds; one such internationally recognised area is Saunders Island, located off the coast of the West Falkland. It contains breeding populations of gentoo penguins (Pygoscelis papua), Magellanic penguins (Spheniscus magellanicus), black-browed albatross (Thalassarche melanophris), king penguins (Aptenodytes patagonicus) and western rockhopper penguins (Eudyptes chrysocome chrysocome). The fungal communities of these sites were investigated using a fungal metabarcode approach. We found these sites contain a large spatial heterogeneity, with communities that are dominated by saprotrophic fungi. However, we noticed that the most abundant species tended to be known mycoparasites. We hypothesise the fungal communities in system undergo a 'boom-bust' cycle of varying alpha diversity which is dependent upon the breeding populations and driven by highly competitive mycoparasitic fungi.

Keywords

Metabarcoding, Falkland Islands, Seabirds, Fungi, High-throughput sequencing, community ecology

* Corresponding author: p.vanwest@abdn.ac.uk 
Introduction

Soil microbial communities are complex and heterogeneous (Zinger et al. 2011; Barberan et al. 2014). With the advent of modern molecular biological resources and techniques, microbial community studies (including fungi) have transitioned from solely culture based approaches (Durrell \& Shields 1960) to PCR based (Viaud et al. 2000). More recently, high throughput sequencing is a procedure that has become increasingly ubiquitous in studies of microbial ecology, with metabarcoding being a commonly used technique (Jumpponen \& Jones 2009; Ghannoum et al. 2010; Buée et al. 2014).

These techniques have been applied using substrates such as soil and dung, in the context of ecological study of vertebrates, to places in the Southern Hemisphere such as Antarctica (Del Frate \& Caretta 1990; Zdanowski et al. 2004; Arenz \& Blanchette 2011). Zdanowski et al. (2004) used culture and molecular techniques to investigate the bacterial composition of Adélie penguin (Pygoscelis adeliae) guano, obtaining 72 colonies in pure culture from King George Island, Antarctica. Similar work was carried out by Del Frate \& Caretta (1990) to isolate fungal cultures from soil, guano and feathers of various bird species, including penguins, isolating 122 colonies of 15 fungal species across various sites in Antarctica. More recently, Arenz and Blanchette (2011) conducted a culture based meta-analysis of soil collected across Antarctic ice-free areas, successfully isolating 455 fungal colonies. Their conclusions noted the importance of Cadophora and Geomyces spp. as diverse and widespread generalist decomposers (Arenz \& Blanchette 2011). Additionally, culture based investigations by Abneuf et al. (2016), investigating the antimicrobial activity of microfungi from Antarctic soil have isolated various different phyla of fungi including Geomyces sp., Mucor sp. and many anamorphic fungi (Abneuf et al. 2016). Their conclusions implicated noteworthy levels of antimicrobial activity in many isolated strains, with anti-bacterial compounds seemingly more prevalent than anti-fungal (Abneuf et al. 2016). 
Fungi more generally have a very complex and important relationship with ecological systems, providing benefits to natural and agricultural systems through mycorrhizal interactions (Berg 2009; Bahram et al. 2015), pathogenic (McCartney et al. 2003) and saprotrophic interactions (Hobbie et al. 2001), with grey areas of overlapping trophic lifestyles amongst certain fungal genera (Nguyen et al. 2016). Tedersoo et al. (2014) attempted to map global biogeography of soil fungi, using a metabarcode approach. Their study indicated fungi have a spatially patterned distribution, with endemic and cosmopolitan distributions. More recently, focus has been growing on the potential of taxonomic assignments, made from metabarcode data, to predict the guild (or function) of individual members and, more importantly, community functional assemblages, although this is in relative infancy (Nguyen et al. 2016).

The Falkland Islands lie north of Antarctica, and north of the Polar Front (the boundary of two air masses at approximately $60^{\circ}$ latitude, arising as a result of cold polar air meeting warmer air); the climate is classed as cool oceanic (Wilson et al. 1993) with average temperatures that vary from 9.4 ${ }^{\circ} \mathrm{C}$ in summer to $2.2{ }^{\circ} \mathrm{C}$ in winter. Precipitation is greater on the east side of the archipelago with the annual precipitation in the capital, Stanley, is $640 \mathrm{~mm}$, but this can vary from 400 to $800 \mathrm{~mm} \mathrm{y}^{-1}$ (McAdam 1993).

Saunders Island (SI) is the second largest offshore island in the Falklands and is internationally recognised as an Important Bird Area (FK14). It contains significant breeding populations of gentoo penguins (Pygoscelis papua), Magellanic penguins (Spheniscus magellanicus), black-browed albatross (Thalassarche melanophris), king penguins (Aptenodytes patagonicus) and western rockhopper penguins (Eudyptes chrysocome chrysocome), as well as other bird species (Otley et al. 2008). Despite the importance of SI to numerous breeding populations there have been no attempts to investigate the microbiota inhabiting these areas. The aim of this study is to characterise the fungal communities of the 5 aforementioned seabirds' nest sites using a metabarcoding approach to assess the inter-and intra-site variation between nest sites and the basal fungal community of SI soil. With 
this, the aim is to use the relatively new FUNGuild analysis to infer the function of these communities.

2. Methods

\subsection{Sample Collection and Storage}

All samples were collected on 11/02/2017 at Saunders Island, Falkland Islands. The nest sites selected belonged to gentoo penguins (Pygoscelis papua) (51 $\left.18^{\circ} 27.17^{\prime \prime} \mathrm{S}, 60^{\circ} 14^{\prime} 14.90^{\prime \prime} \mathrm{W}\right)$, Magellanic penguins (Spheniscus magellanicus) (5118'31.67"S, 60¹4'11.30"W), black-browed albatross (Thalassarche melanophris) (51ำ $\left.18^{\prime} 26.83^{\prime \prime S}, 60^{\circ} 13^{\prime} 19.63^{\prime \prime} \mathrm{W}\right)$, king penguins (Aptenodytes patagonicus) (51 $\left.11^{\circ} 28.52^{\prime \prime S}, 60^{\circ} 14^{\prime} 0.62^{\prime \prime} \mathrm{W}\right)$ and western rockhopper penguins (Eudyptes chrysocome chrysocome) $\left(51^{\circ} 18^{\prime} 27.09^{\prime \prime} \mathrm{S}, 60^{\circ} 13^{\prime} 36.13^{\prime \prime} \mathrm{W}\right)$. A control site near to the main collection of penguin nests was also chosen $\left(51^{\circ} 18^{\prime} 30.67^{\prime \prime} S, 60^{\circ} 14^{\prime} 10.98^{\prime \prime} \mathrm{W}\right)$, which represented an area with heavily sheep-grazed vegetation. The sites selected are shown graphically in Figure 1.

Signs of bird presence, such as droppings and feathers, were not detected at the site of the control sample. Collection of soil samples was carried out ensuring stress to animals would be minimal. At each site a GPS record was taken and 3 individual soil samples were collected, the depth was kept roughly the same (full accuracy was impossible due to the very sandy nature of most of the samples) and all samples in each site were collected in no more than a $2 \mathrm{~m}$ radius. Basic information about each site was also recorded, such as chick/adult numbers, the basic soil qualities and any other relevant information. The samples were kept cool post-collection; and were homogenised and saturated with LifeGuard Soil Preservation Solution (MO BIO) within $12 \mathrm{~h}$ of collection and kept at $20{ }^{\circ} \mathrm{C}$ except for intermittent times of travel and during the DNA extraction process. 


\subsection{Sample Metabarcoding}

The DNA was extracted using the DNeasy PowerSoil Kit (Qiagen), the protocol was followed but during the lysis step a FastPrep (MP) machine was used with three replicates of $5 \mathrm{~m} / \mathrm{s}$ for 45 seconds. The remaining metabarcoding protocol was carried out at the Centre for Genome-Enabled Biology and Medicine (University of Aberdeen). PCR reactions were performed on a Life Technologies Veriti thermal cycler (Thermo Fisher Scientific) with the a reaction volume of $25 \mu \mathrm{l}$ comprised of $2.5 \mu$ l of DNA, $5 \mu$ l each of $1 \mu \mathrm{M}$ forward and reverse primer (ITS1F CTTGGTCATTTAGAGGAAGTAA, ITS1R GCTGCGTTCTTCATCGATGC), $12.5 \mu$ l KAPA HiFi HotStart ReadyMix 2X (Kapa Biosystems) with 15 cycles of PCR ( $95^{\circ} \mathrm{C}$ for 3 min; 15 cycles of: $95{ }^{\circ} \mathrm{C}$ for $30 \mathrm{sec}$, $55^{\circ} \mathrm{C}$ for $30 \mathrm{sec}, 72{ }^{\circ} \mathrm{C}$ for $30 \mathrm{sec} ; 72{ }^{\circ} \mathrm{C}$ for $5 \mathrm{~min}$, and a final hold at $4{ }^{\circ} \mathrm{C}$ ). Three reactions were performed for each sample to reduce the potential for PCR bias.

The pooled reactions were purified with AMPure XP (Beckman Coulter) according to manufacturer protocol with an AMPure XP to sample ratio of $0.8 x$. The purified amplicons containing the Illumina overhang were indexed with Illumina Nextera XT v2 indices (Illumina). Briefly, the dual indexed Illumina libraries were prepared with $5 \mu$ l of DNA, $5 \mu$ leach of i5 and i7 index primer, $25 \mu$ l KAPA HiFi HotStart ReadyMix, and $10 \mu \mathrm{l}$ of PCR grade water with 8 cycles of PCR $\left(95^{\circ} \mathrm{C}\right.$ for $3 \mathrm{~min} ; 8$ cycles of: 95 ${ }^{\circ} \mathrm{C}$ for $30 \mathrm{sec}, 55^{\circ} \mathrm{C}$ for $30 \mathrm{sec}, 72{ }^{\circ} \mathrm{C}$ for $30 \mathrm{sec} ; 72{ }^{\circ} \mathrm{C}$ for $5 \mathrm{~min}$, and a final hold at $4{ }^{\circ} \mathrm{C}$ ) on a Life Technologies Veriti thermal cycler. The indexed libraries were purified with AMPure XP with an AMPure XP to sample ratio of 0.9x.

Libraries were quantified using the Thermo Fisher Scientific Quant-iT ${ }^{T M}$ dsDNA High Sensitivity Assay and the fluorescence measured on a BMG Labtech FLUOstar Omega microplate reader (BMG Labtech). The quality and size in base pairs of the libraries were analysed on an Agilent 2200 TapeStation with High Sensitivity D1000 ScreenTapes (Agilent Technologies).

The libraries were equimolar pooled and sequenced on an Illumina MiSeq Sequencing System using MiSeq v3 chemistry with 300 bp paired-end reads. Base calling and fastq output files were generated 
with RTA software on the MiSeq instrument.

\subsection{Preparing Metabarcode Data}

The data were processed using PIPITS (Gweon et al. 2015) using the standard settings, with Internal Transcribed Spacer 1 (ITS1) selected as the region sequenced. The paired-end reads were joined using PEAR (Zhang et al. 2014) and ambiguous sequences removed using FASTX (Hannon, http://hannonlab.cshl.edu); the ITS1 region was extracted from each sequence using ITSx (Bengtsson-Palme et al. 2013). The Operational Taxonomic Units (OTUs) were generated by clustering the sequences to $97 \%$ similarity using VSearch (Rognes, https://github.com/torognes/vsearch) and chimeric sequences were removed using the UNITE UCHIME reference database. Finally the OTUs were given a taxonomic assignment using RDP Classifier (Wang et al. 2007) trained with a UNITE fungal ITS reference training data set (Gweon et al. 2015). Once the OTU list was compiled the sequence data was checked first using a locally created BLAST database, accepting taxonomic assignment matches of $>97 \%$ and gaps of $<2 \%$. Secondly the UNITE BLAST database was used for all unmatched sequences, accepting matches with an E-value of $1 \times 10^{-24}$ or lower. Finally all sequences remaining unmatched were checked with NCBI BLAST, results with an E-value of $1 \times 10^{-24}$ and lower were considered when modifying or adding to the taxonomic assignment, singleton BLAST results were not considered. Any OTUs with unsuccessful assignments still present were labelled unassignable.

\subsection{Alpha and Beta Diversity Calculations}

The subsequent in silico analysis was done using the QIIME1 platform (Caporaso et al. 2010). The Good's Coverage was checked to ensure sampling depth was sufficient, one sample of the three soil samples from the western rockhopper nest had a poor total sequence count and was removed. All 
remaining samples were subsampled to 30,000 sequences. The alpha diversity of each sample was calculated using the Chao and Shannon metrics which were then compared with other samples from the same site by taking the mean and $95 \%$ confidence interval, the beta diversity was calculated using the Bray-Curtis similarity resemblance measure. The beta diversity calculation was used to create two Principle Coordinates Analysis (PCoA) plots, displaying PCoA 1 and 2 and PCoA 1 and 3. ANOSIM tests were carried out on the Bray-Curtis measure to check for any significant correlations between all sites and between the nest and control sites as well as any clusters seen in the analysis.

\subsection{Procrustes Analysis}

A Procrustes analysis was carried out to investigate any potential relationship between bird diets and fungal diversity in the nests. The diets of each bird, king penguin (Cherel et al. 2002a), gentoo penguin, Magellanic penguin, rockhopper penguin (Clausen \& Pütz 2002) and black-browed albatross (Cherel et al. 2002b)) were collated and dissimilarity calculated using Bray-Curtis measure. This Bray-Curtis measure was used to create a PCoA plot. This plot was used with a Bray-Curtis PCoA plot generated using the subsampled OTU table that had the control samples removed and each site's OTUs combined. The analysis was carried out with QIIME1 with 1000 permutations to calculate a Monte Carlo $\left(\mathrm{M}^{2}\right)$ value (Caporaso et al. 2010).

\subsection{Comparing Fungal Communities of Nest Sites to Control}

To investigate the community composition of each site, comparing specifically the nests against the control sites; the subsampled OTU table was used, all singletons and non-fungal OTUs were removed. The OTUs were grouped into 3 clusters and counted, those retrieved from only the control site, those present in both control and nests and those solely in nests. The class percentage composition of OTUs which had a class assignment were calculated in each subgroup to visualise any 
differences. A Venn diagram was created to visualise the three subgroups using VennDiagram on $R$ (https://cran.r-project.org/package=VennDiagram).

\subsection{Visualising Dominant Species by Site}

To visualise the most abundant species in each site, the subsampled OTU table was used. All nonfungal OTUs were removed and the individual samples from each site were combined. The percentage abundance of each OTU was calculated by dividing sequence counts of that OTU against total sequence count in that site $(90,000$ for all except Rockhopper $(60,000))$. The species were taken from the BLAST-checked taxonomy table, the highest taxonomic level successfully assigned was used. The 10 most abundant species and the percentage of all remaining species at each site were used to create a bar chart.

\subsection{FUNGuild Analysis}

Finally, to parse the fungal community dataset from the subsampled OTU table into functional groups (or guilds) the online resource FUNGuild was used (Nguyen et al. 2016). The FUNGuild software annotates taxonomic data within the OTU table with corresponding data on its online database, the annotations include the guild, trophic mode and growth morphology; only confidence scores of 'Probable' and 'Highly Probable' were used. The individual samples in the annotated table were pooled by site for analysis and the sequence counts of each OTU were used for analysis of FUNGuild annotations. 


\section{Results}

Rudimentary information about the soil samples collected are shown in Table 1, when the samples were collected chicks from all different species were still present. In the case of gentoo the chicks had moved from their nest site and had lost virtually all their down feathers. The same was true of the Magellanic penguin and rockhopper penguin chicks. Only the king penguin and albatross chicks had all down feathers present.

The soil samples themselves varied widely from wet to very dry and had varying colours indicating different levels of organic compounds within them. The extracted DNA had different concentrations but all were successfully amplified during the PCRs.

The number of raw reads for all sites was approximately $1.4 \times 10^{6}$, using PIPITS this was translated to 2727, $97 \%$ clustered operational taxonomic units (OTUs). Although Good's Coverage predicted species coverage to be $>99 \%$ for all samples, the total number of sequences achieved for sample Rockhopper $2(\mathrm{RH} 2)$ was low compared to all other samples and was therefore removed from further analysis to prevent large decreases in Good's Coverage when the OTU table was subsampled. After subsampling to 30,000 sequences per site the Good's Coverage was retained at $>99 \%$.

\subsection{Alpha Diversity}

The alpha diversity analysis (Figure 2) showed large inter-site differences, with smaller but significant levels of intra site differences in certain samples, with the Chao 1 species richness estimator value ranging from 190 species in Rockhopper penguins $(\mathrm{RH})$ to 805 species with Magellanic penguins (Mag), indicating large population differences. Mag and RH also had the highest and lowest Shannon index values, of 6.22 and 2.25 respectively.

Although Mag had the largest species richness this was only significantly different using the Shannon metric, the site Albatross (Alb) had a similar Chao 1 value to Mag, suggesting the number of species 
is similar but Mag has greater evenness of species. The control (Con) samples showed relatively low variation from the mean using both metrics when compared to the nest samples. Sites gentoo penguins (Gen) and king penguins (King) had smaller confidence intervals using the Chao 1 index than the Shannon index in which Gen had the largest $95 \%$ confidence interval seen.

\subsection{Beta Diversity}

The beta diversity principle coordinate analysis ( $\mathrm{PCOA}$ ) plots (Figure 3) were created using the BrayCurtis dissimilarity matrix, with plot (a) showing principle coordinate (PCOA) 1 charted against PCoA 2. In (b) PCOA 1 was charted against PCoA 3; they both contained $42.9 \%$ and $39.8 \%$ of the total variation respectively seen in the OTU table. In total all 3 principle coordinates shown in Figure 3 explained $57.3 \%$ of the total variation.

The PCoA charts in Figure $\mathbf{3}$ charted the principal coordinates, detailing the dissimilarity between samples, which indicated a clustering of individual samples within each site (with Alb3 being a notable outlier). ANOSIM analyses, comparing the intra-site similarity using the beta diversity data indicated a strong positive correlation of beta diversity groupings within sites compared to between sites (ANOSIM, $R=0.843, p=0.001$, permutations $=999$ ). The clustering in Figure 3 (especially in $(b)$ ) indicated there could be a difference between the nest sites compared to the control, ANOSIM analysis showed there to be a weak statistically significant greater similarity between nest sites and between control sites (ANOSIM, R $=0.286, p=0.021$, permutations $=999$ ). Smaller clusters of sites Mag and Alb and sites Gen and RH were also seen, analysing using ANOSIM showed there to be no significant differences between cluster Mag/Alb and all other sites (ANOSIM, R $=-0.043, p=0.641$, permutations $=999)$ while cluster Gen/RH had a significant similarity compared to all other sites (ANOSIM, R = 0.599, $p=0.001$, permutations $=999$ ). The site King also seemed to cluster independently from all the other sites, however ANOSIM analysis indicated only a small degree of non-significant similarity from all other samples (ANOSIM, R = 0.127, $p=0.118$, permutations $=999$ ). 


\subsection{Procrustes Analysis}

The Procrustes analysis compared a combined site (control removed) OTU table Bray-Curtis PCoA plot against a Bray-Curtis PCoA plot which was derived from diet information of all bird species whose nest sites are in this investigation. The diet species labels were combined with the OTU site labels (i.e. King_OTU_PCoA and King_diet_PCOA) and the Procrustes script attempted to find a significant correlation between the diet and OTU table. The resultant permutated analysis indicated no correlation, indicated by the high $\mathrm{M}^{2}$ however this was not statistically significant $\left(\mathrm{M}^{2}=0.777, \mathrm{p}=\right.$ 0.473 , permutations $=999$ ) .

\subsection{Fungal Community Comparison of Nest Sites against Control Sites}

The OTUs which were assigned to fungi were used to compare the fungal diversity of the control sites and nest sites (Figure 4), this was to investigate if community structures were different depending on if they were present in the control site, nest site(s) or both. There was a large overlap between the nest sites and control sites in terms of fungal OTU counts (Figure 4). While there were 158 unique OTUs in the control site there were 1297 OTUs not present in the control site which corresponds to $\sim 259$ non-control OTUs on average in each nest site. The data also showed there were 47 cosmopolitan OTUs which were found in every site including the control.

The dominant classes changed depending on their site location, in the control site the dominant class was Dothideomycetes (27\%) which decreased to $16 \%$ in the both subgroup, and in the nest subgroup decreased to $12 \%$ which represented a drop two places in the most abundant classes

(Figure 4). The reciprocal result was seen in the nest subgroup with the Sordariomycetes being the most dominant class at $21 \%$ before they decreased to $14 \%$ in the both subgroup and finally decreased to $12 \%$ in the control subgroup having dropped to the $4^{\text {th }}$ most common fungal class. 
Interestingly, certain classes stayed relatively constant, the Leotiomycetes only varied by $2 \%$ across all subgroups.

\subsection{Inter-site Fungal Abundances}

The most dominant species from each site was calculated using the abundance data from the subsampled OTU table (Figure 5). The highest successfully assigned rank was used to label each OTU. The Gen chart shows one species, Debaryomyces hansenii, dominated the site, and represented just under half of all ITS sequences generated from that site. Similarly, such dominances were seen in site King (Sporidiobolus pararoseus), Rockhopper (Penicillium rubidurum) and to a lesser extent the control site (Vishniacozyma victoriae). The sites Magellanic and Albatross did not show this individual dominance, instead they had a more even abundance of OTUs. The Gen site also had a relatively large number of most abundant OTUs with only a kingdom Fungi assignment compared to all other sites, at 5 .

\subsection{FUNGuild Functional Predictions}

The taxonomic data from the subsampled OTU table was used for the FUNGuild annotations. Which made predictions of the functional attributes of each of the OTUs. The nest sites all displayed the same trend, with saprotrophic fungi being the most abundant, and the majority of remaining fungi having pathogenic trophic modes (Figure 6). Mag and Alb differed slightly as they contained a small percentage of symbiotrophic fungi. The control site had a very different trophic makeup, containing a large proportion of symbiotrophic fungi ( $36 \%$ ) as well as a large proportion of primarily pathotrophic fungi, with primarily saprotrophic fungi accounting for just $12 \%$. The proportion of successfully assigned sequences across the sites varied widely. There was no site that achieved $>50$ \% sequence annotation, site Mag have $48 \%$ assigned, while site Gen and RH had the lowest at $14 \%$. 
Discussion

Overall the data we received from the metabarcoding approach showed a suitable sequence depth; despite having to remove one sample from the analysis.

The alpha diversity (Figure 2) across all the sites showed significant differences, indicating that the sites have a range of differing abundances and evenness's. Sites Mag and Alb, and to a lesser extent Con had larger community membership, with Mag having overall the greatest evenness (reflected similarly in Figure 5). Because the control site showed relatively low variation from the mean, taking both Chao 1 and Shannon metrics into account, this suggests perhaps a more stable number of species and community evenness. In contrast, sites Gen and King had lower alpha diversity values, suggesting those two sites had a smaller, yet stable community membership, but had highly uneven population sizes (reflected in Figure 5).

The beta diversity plots (Figure $\mathbf{3}$ ) explained $57.3 \%$ of the total variation. The individual samples for the most part clustered with respect to their site. This is perhaps not unsurprising given the similar environment in each nest site and much lesser geographical distance between intra-site samples. There was also a significant clustering of nest sites against the control, indicating a slightly greater dissimilarity between nest site samples and the control, which is also supported by our findings described (Figure $\mathbf{5}$ and $\mathbf{6}$ ) with different highly abundant species and community trophic strategy. The clustering seen with sites Gen and RH compared to all other samples suggests those nest sites share a characteristic in terms of their beta diversity which is unique to all others; although what this is precisely, is unknown.

The nesting seabirds' diets were also used to check if a correlation with fungal assembly and richness could be found. The Procrustes analysis indicated there was no biologically relevant correlation between the penguin diets and the fungal communities, this result was also not significant. We 
postulate that despite the negative result, diet could still be having an impact, but is being muted by other factors such as type of soil and vegetation (or lack thereof) present on the respective sampling sites.

As previously mentioned, there was a significant difference in fungal community between the control site and the nest sites. Figure 4 demonstrates clearly a membership change between the control and nest OTUs, which is supported by the intermediate shared OTUs acting like a 'stepping stone' between the nest and control, this trend can be seen in the Dothideomycetes, Tremellomycetes and Eurotiomycetes. Notably there was little membership change in the Leotiomycetes, suggesting that members of that class have functions that are not affected by the nesting birds.

There was a clear difference between the most abundant OTUs in each site. Group GKR (Gen; King \& RH) had one clearly dominant species each (Debaryomyces hansenii, Sporidiobolus pararoseus and Penicillium rubidurum, respectively) while group AM (Alb \& Mag) did not. These dominant species are all able to grow in harsh conditions and all have been investigated as potential mycoparasites (Sharma et al. 2008; Kusari et al. 2013; Banjara et al. 2016). Very little work has been carried out on P. rubidurum, whereas there has been extensive work done on on D. hansenii, and interestingly increased $\mathrm{NaCl}$ concentration is associated with enhanced killing action in this species (Llorente et al. 1997). With the coastal nature of the sampling area, increased salt concentration would be expected compared to inland. An increased "killer"-phenotype could explain D. hansenii's success in many of these areas.

All nesting sites (except for King) have intermittent periods throughout the year where the bird populations are not present. Mycoparasite domination in many of these sites suggests that they could have adaptations which allow them to thrive in the absence of bird nesting; as mycoparasites, they have the potential to outcompete other fungi, perhaps even deriving energy from fellow fungi. In sites Gen and RH the penguin chicks were very close to fledging and in the case of Gen they had 
already left the nest site and had formed crèches close to the sea. Site King also had a smaller population, with smaller evidence of droppings in the site that we sampled, indicating their activities introduces fewer energy sources into the soils. Contrastingly sites Alb and Mag were both being actively used and they had the communities with the largest Shannon index.

Despite the large differences in community evenness, when the functional attributes of each site were investigated using FUNGuild (Figure 6), all nest sites had a very similar functional assemblage in comparison to the control site, being dominated by saprotrophic fungi, with pathogenic associated fungi representing the large remainder of functional diversity. In contrast the control site had a much larger symbiotrophic membership and a relatively small saprotrophic one, suggesting that overall, the fungi in the control site are carrying out a greater amount of non-saprotrophic functions.

The functional similarities in all nest sites, despite the intermittent seabird presence in most sites, could possibly be due to a significant degree of functional dormancy in which, despite conditions being unfavourable in some sites they retained their function in a dormant state of metabolic stasis. It would be logical to suggest that during these periods, interspecies competition for limiting resources would be occurring, possibly explaining the high abundance of mycoparasites.

To conclude, our results indicate that the fungal communities in this region show spatial heterogeneity with far greater homogeneity within the nest sites. This spatial heterogeneity is in line with previous studies into soil fungal diversity (Jumpponen \& Jones 2009; Tedersoo et al. 2014). There is evidence to suggest the control site has a greater degree of temporal stability, perhaps because the nest site communities suffer the added ecological stress of going through periods where birds are absent. Despite some samples showing much greater species richness, the predicted function remained constant. A potential explanation for our results could be that there is a 'boombust' cycle of fungal diversity which is driven largely by highly competitive mycoparasites in response to the seabird's cyclical presence and absence in these sites. 
Acknowledgements

Our work is supported by the BBSRC (BB/M010996/1, BB/N005058/1, BB/P020224/1) (JH, PvW), NERC (NE/P007570/1) (PVW), The South Atlantic Environmental Research Institute (SAERI) (JH) and the University of Aberdeen (PvW). We would like to thank the Pole-Evans family for giving us permission to sample on their land.

References

Abneuf, M.A. et al., 2016. Antimicrobial activity of microfungi from maritime Antarctic soil. Czech Polar Reports, 6(2), pp.141-154. Available at: http://www.sci.muni.cz/CPR/LP622016/LP-6_213.htm.

Arenz, B.E. \& Blanchette, R.A., 2011. Distribution and abundance of soil fungi in Antarctica at sites on the Peninsula, Ross Sea Region and McMurdo Dry Valleys. Soil Biology and Biochemistry, 43(2), pp.308-315. Available at: http://dx.doi.org/10.1016/j.soilbio.2010.10.016.

Bahram, M., Peay, K.G. \& Tedersoo, L., 2015. Local-scale biogeography and spatiotemporal variability in communities of mycorrhizal fungi. New Phytologist, 205(4), pp.1454-1463.

Banjara, N. et al., 2016. Killer toxin from several food-derived Debaryomyces hansenii strains effective against pathogenic Candida yeasts. International Journal of Food Microbiology, 222, pp.23-29. Available at: http://dx.doi.org/10.1016/j.ijfoodmicro.2016.01.016.

Barberan, A. et al., 2014. Why are some microbes more ubiquitous than others? Predicting the habitat breadth of soil bacteria J. Klironomos, ed. Ecology Letters, 17(7), pp.794-802. Available at: http://doi.wiley.com/10.1111/ele.12282.

Bengtsson-Palme, J. et al., 2013. Improved software detection and extraction of ITS1 and ITS2 from ribosomal ITS sequences of fungi and other eukaryotes for analysis of environmental sequencing data M. Bunce, ed. Methods in Ecology and Evolution, 4(10), pp.914-919. Available 
at: http://doi.wiley.com/10.1111/2041-210X.12073 [Accessed August 30, 2017].

Berg, G., 2009. Plant-microbe interactions promoting plant growth and health: Perspectives for controlled use of microorganisms in agriculture. Applied Microbiology and Biotechnology, 84(1), pp.11-18.

Buée, A.M. et al., 2014. 454 Pyrosequencing analyses of forest soils reveal an high fungal diversity unexpectedly. New Phytologist, 184(2), pp.449-456.

Caporaso, J.G. et al., 2010. QIIME allows analysis of high-throughput community sequencing data. Nature Methods, 7(5), pp.335-336. Available at: http://www.nature.com/doifinder/10.1038/nmeth.f.303 [Accessed August 31, 2017].

Cherel, Y., Pütz, K. \& Hobson, K.A., 2002. Summer diet of king penguins (Aptenodytes patagonicus) at the Falkland Islands, southern Atlantic Ocean. Polar Biology, 25(12), pp.898-906. Available at: http://www.cebc.cnrs.fr/publipdf/2002/CPB25.pdf [Accessed September 21, 2017].

Cherel, Y., Weimerskirch, H. \& Trouve, C., 2002. Dietary evidence for spatial foraging segregation in sympatric albatrosses ( Diomedea spp.) rearing chicks at lles Nuageuses, Kerguelen. Marine Biology, 141(6), pp.1117-1129. Available at:

http://www.cebc.cnrs.fr/publipdf/2002/CMB141.pdf [Accessed September 22, 2017].

Clausen, A.P. \& Pütz, K., 2002. Recent trends in diet composition and productivity of gentoo, magellanic and rockhopper penguins in the Falkland Islands. Aquatic Conservation: Marine and Freshwater Ecosystems, 12(1), pp.51-61. Available at: https://www.researchgate.net/profile/Klemens_Puetz/publication/227828951_Recent_trends _in_diet_composition_and_productivity_of_Gentoo_Magellanic_and_Rockhopper_Penguins_i n_the_Falkland_Islands/links/02bfe5128ad1f09910000000.pdf [Accessed September 21, 2017].

Durrell, L.W. \& Shields, L.M., 1960. Fungi Isolated in Culture from Soils of the Nevada Test Site. Mycologia, 52(4), pp.636-641. Available at: http://www.jstor.org/stable/3756096 [Accessed 
September 26, 2017].

Del Frate, G. \& Caretta, G., 1990. Fungi isolated from Antarctic material. Polar Biology, 11(1), pp.1-7.

Ghannoum, M.A. et al., 2010. Characterization of the oral fungal microbiome (mycobiome) in healthy individuals. PLoS Pathogens, 6(1).

Gweon, H.S. et al., 2015. PIPITS: An automated pipeline for analyses of fungal internal transcribed spacer sequences from the Illumina sequencing platform. Methods in Ecology and Evolution, 6(8), pp.973-980.

Hobbie, E.A., Weber, N.S. \& Trappe, J.M., 2001. Mycorrhizal vs saprotrophic status of fungi: the isotopic evidence. New Phytologist, 150(3), pp.601-610. Available at: http://doi.wiley.com/10.1046/j.1469-8137.2001.00134.x [Accessed September 26, 2017].

Jumpponen, A. \& Jones, K.L., 2009. Massively parallel 454 sequencing indicates hyperdiverse fungal communities in temperate Quercus macrocarpa phyllosphere. New Phytologist, 184(2), pp.438-448.

Kusari, P. et al., 2013. Endophytic fungi harbored in Cannabis sativa L.: diversity and potential as biocontrol agents against host plant-specific phytopathogens. Fungal Diversity, 60(1), pp.137151. Available at: http://link.springer.com/10.1007/s13225-012-0216-3.

Llorente, P. et al., 1997. Effect of salt on the killer phenotype of yeasts from olive brines. Applied and environmental microbiology, 63(3), pp.1165-7. Available at: http://www.ncbi.nlm.nih.gov/pubmed/9055432.

McAdam, J., 1993. Farming on the peatlands of the Falkland Islands,

McCartney, H.A. et al., 2003. Molecular diagnostics for fungal plant pathogens. Pest Management Science, 59(2), pp.129-142.

Nguyen, N.H. et al., 2016. FUNGuild: An open annotation tool for parsing fungal community datasets 
by ecological guild. Fungal Ecology, 20, pp.241-248. Available at:

http://linkinghub.elsevier.com/retrieve/pii/S1754504815000847.

Otley, H. et al., 2008. Falkland Islands State of the Environment Report 2008,

Sharma, R.N., Maharshi, R.P. \& Gaur, R.B., 2008. Sporidiobolus pararoseus Fell \& Tallman - an antagonistic yeast with biocontrol potential. Current Science, 95(8), pp.1003-1004.

Tedersoo, L. et al., 2014. Disentangling global soil fungal diversity. Science, 346(6213), pp.10521053. Available at: http://www.sciencemag.org/cgi/doi/10.1126/science.aaa1185.

Viaud, M., Pasquier, A. \& Brygoo, Y., 2000. Diversity of soil fungi studied by PCR-RFLP of ITS. Mycological Research, 104(9), pp.1027-1032. Available at: http://linkinghub.elsevier.com/retrieve/pii/S0953756208617530.

Wang, Q. et al., 2007. Naive Bayesian Classifier for Rapid Assignment of rRNA Sequences into the New Bacterial Taxonomy. Applied and Environmental Microbiology, 73(16), pp.5261-5267. Available at: http://www.ncbi.nlm.nih.gov/pubmed/17586664 [Accessed August 30, 2017].

Wilson, P. et al., 1993. Soil erosion in the Falkland Islands: an assessment. Applied Geography, 13(4), pp.329-352.

Zdanowski, M.K. et al., 2004. Bacterial diversity in Adelie penguin, Pygoscelis adeliae, guano: molecular and morpho-physiological approaches. FEMS Microbiology Ecology, 50(3), pp.163173. Available at: https://academic.oup.com/femsec/articlelookup/doi/10.1016/j.femsec.2004.06.012.

Zhang, J. et al., 2014. PEAR: a fast and accurate Illumina Paired-End reAd mergeR. Bioinformatics, 30(5), pp.614-620. Available at: https://academic.oup.com/bioinformatics/articlelookup/doi/10.1093/bioinformatics/btt593 [Accessed August 30, 2017].

Zinger, L. et al., 2011. Contrasting diversity patterns of crenarchaeal, bacterial and fungal soil 
communities in an alpine landscape. PLOS ONE, 6(5).

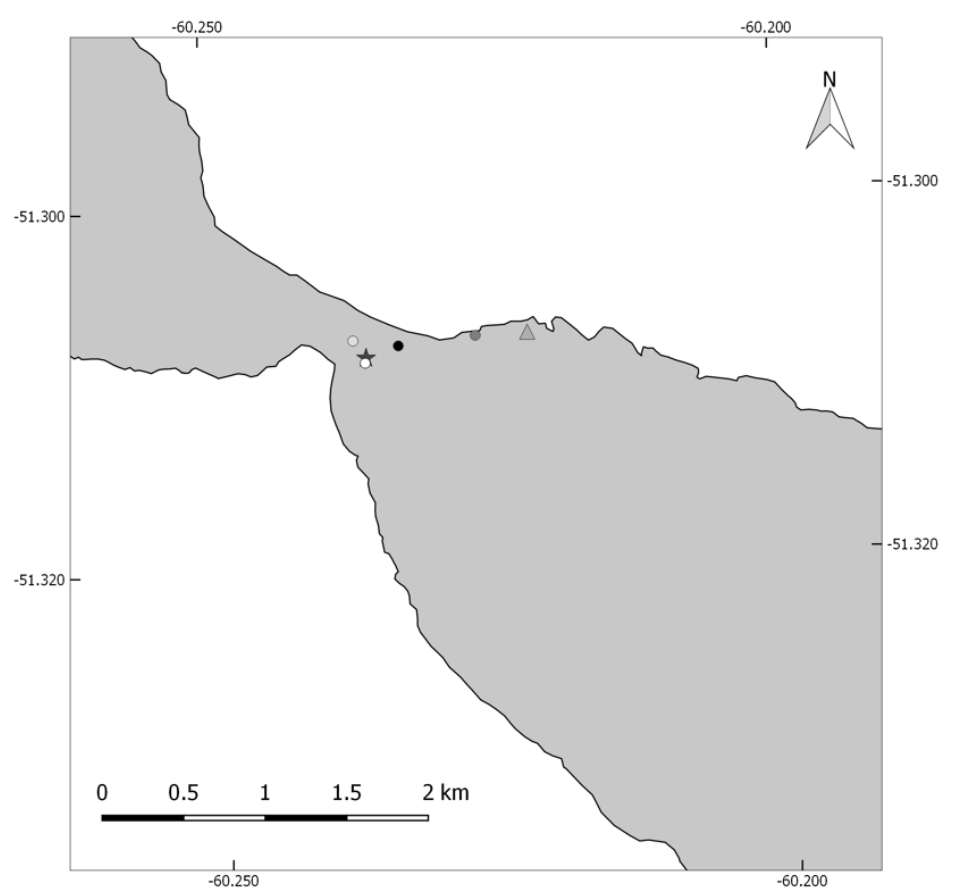

Legend

$\Delta$ Albatross

- Gentoo

- King

Magellanic

- Rockhopper

$\star$ Control

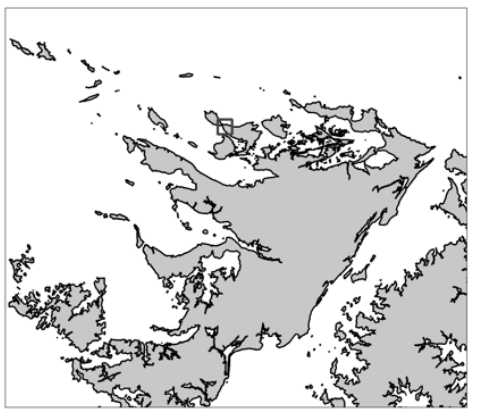

Figure 1 a map of the Neck, Saunders Island, indicating the sites at which the soil samples were collected. 

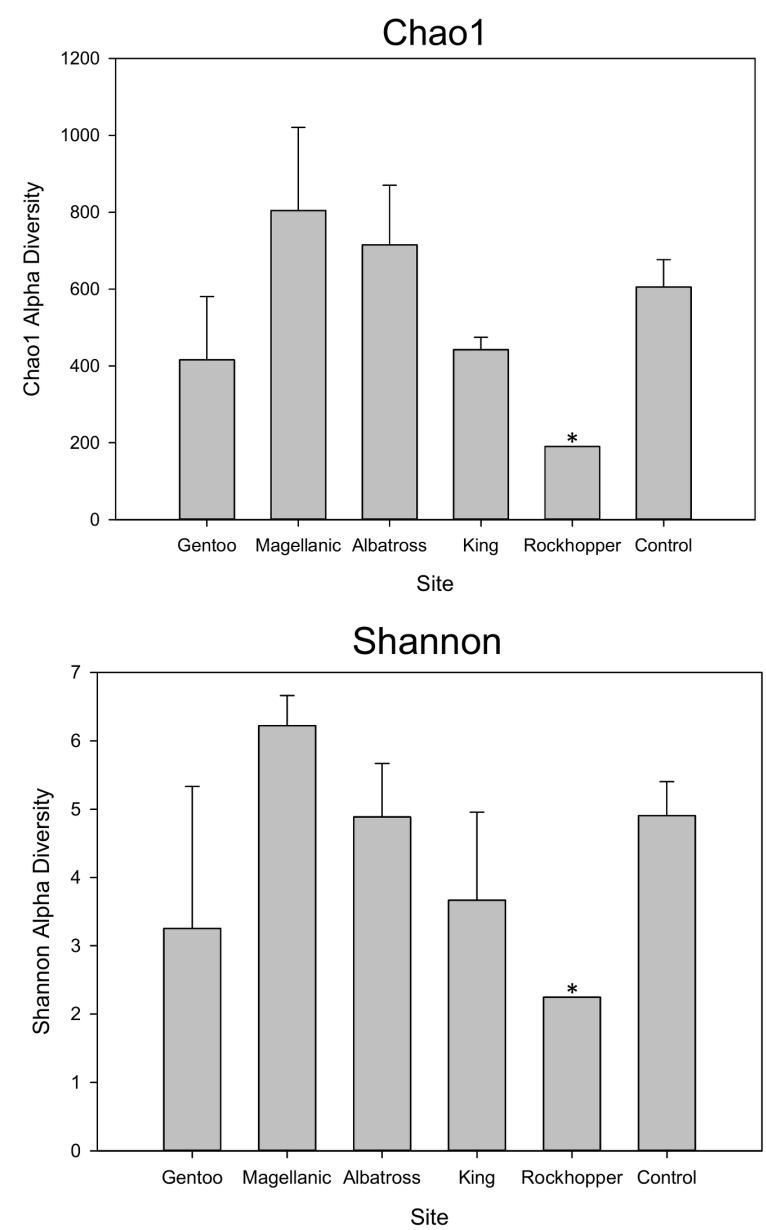

Figure 2 The average Chao 1 index (top) and Shannon index (bottom) of each site, using the 30,000 sequence subsampled OTU table $(n=3$ for all sites apart from rockhopper $(n=2))$. Error bars represent $95 \%$ confidence interval. ${ }^{*}$ No error bars on rockhopper samples due to $\mathrm{n}<3$. 

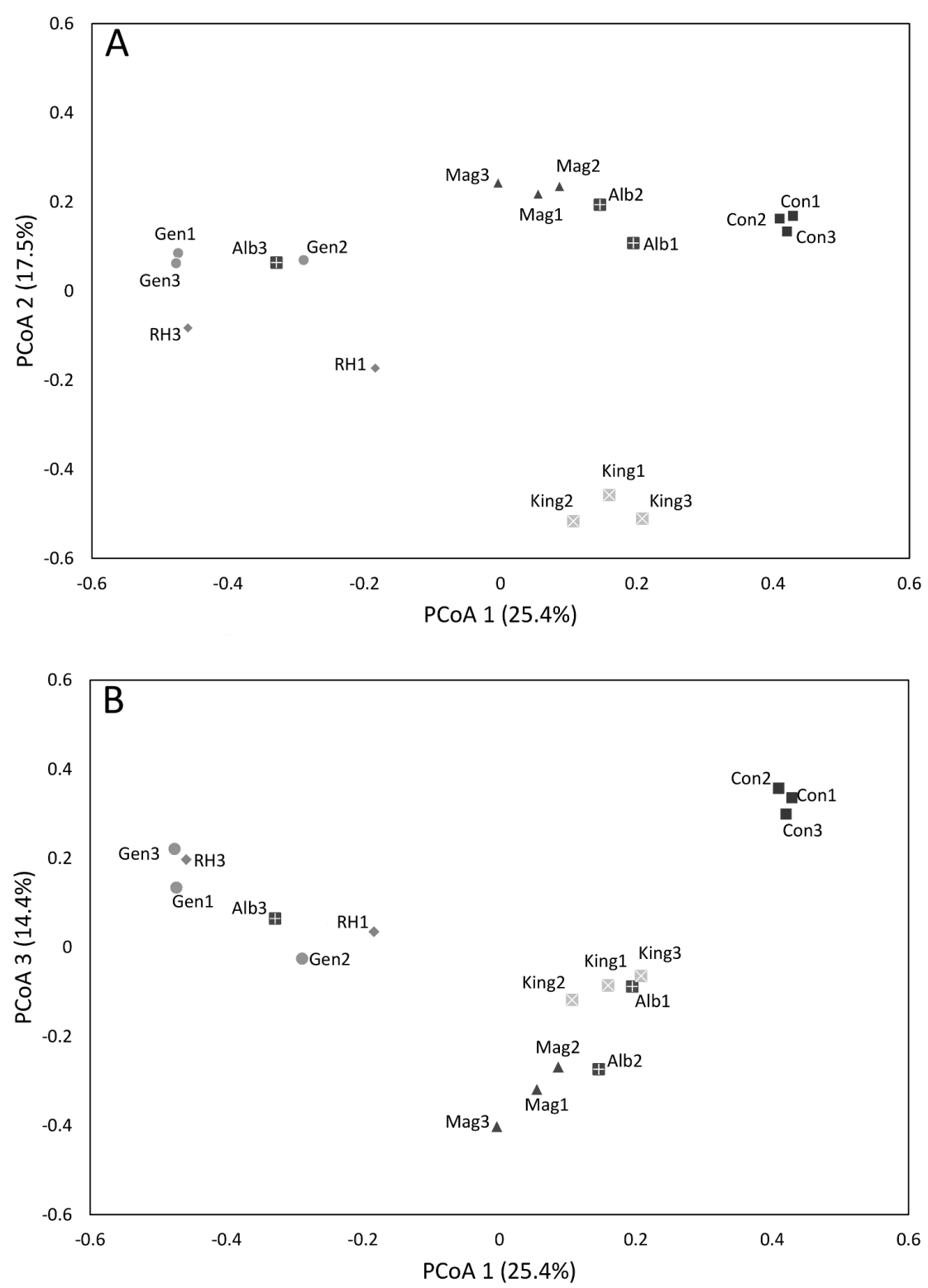

Figure 3 Two PCoA plots of the Bray-Curtis dissimilarity using the $1^{\text {st }}$ and $2^{\text {nd }}$ principle coordinates (a) and the $1^{\text {st }}$ and $3^{\text {rd }}$ principle coordinates (b). The variation explained in plots (a) and (b) is $42.9 \%$ and $39.8 \%$ respectively. Diamonds $=$ rockhopper penguin, triangles $=$ Magellanic penguin, circles $=$ gentoo penguin, dark squares with crosses $=$ albatross, light squares with $x^{\prime} s=$ king penguin and dark squares $=$ Control . 


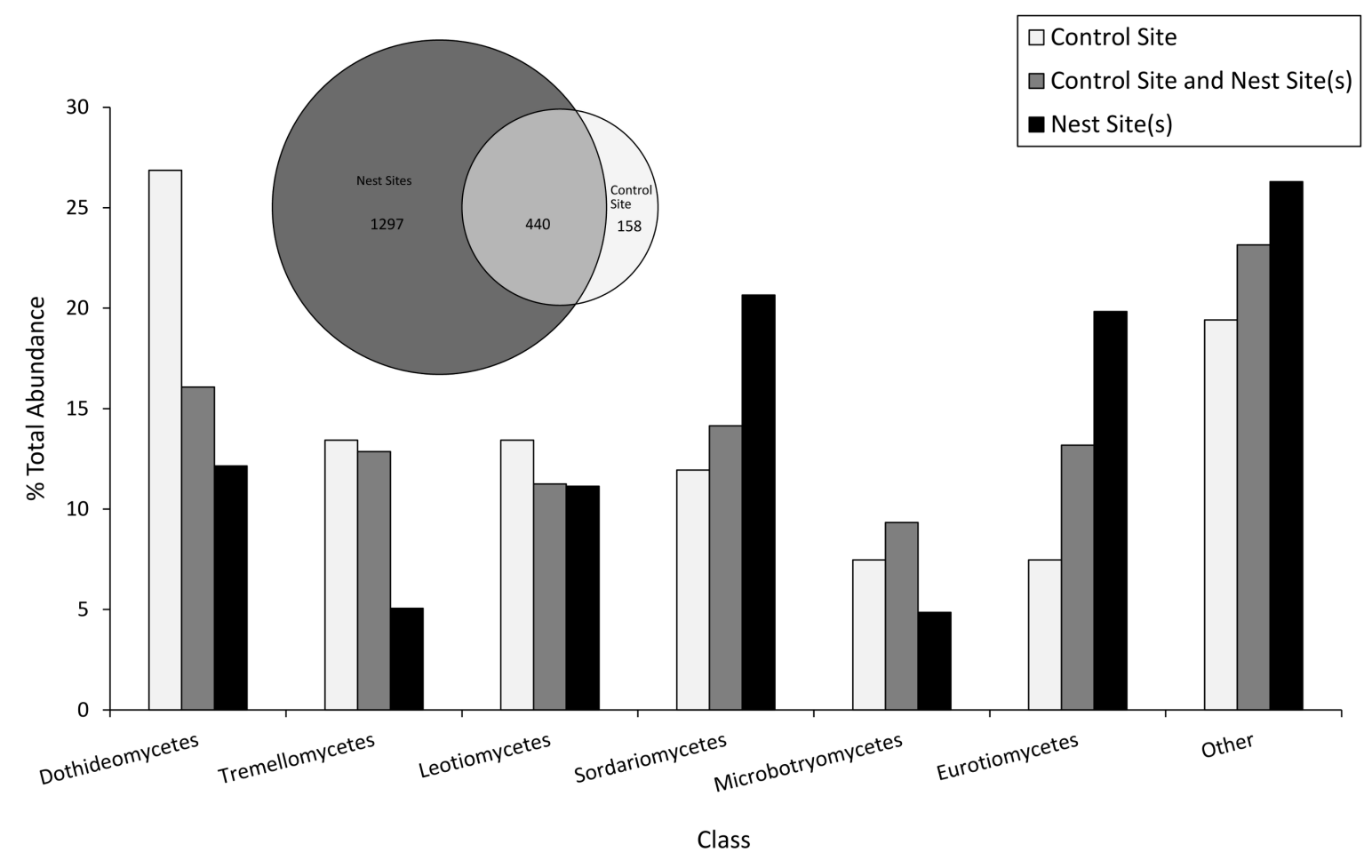

Figure 4 The count of all fungal OTUs from the subsampled OTU table displayed on a Venn diagram characterising them as either present in nest samples (dark grey) (1297 OTUs), present in the control sample (pale grey) (158 OTUs) or present in both (grey) (440 OTUs). The percentage of the most common classes of each group of OTUs is also shown for the control (pale grey), those which are present in both (grey) and those that are found solely in the nest sites (dark grey). For this analysis only OTUs that had class assignments were used ( $84 \%$ of all fungal OTUs). 

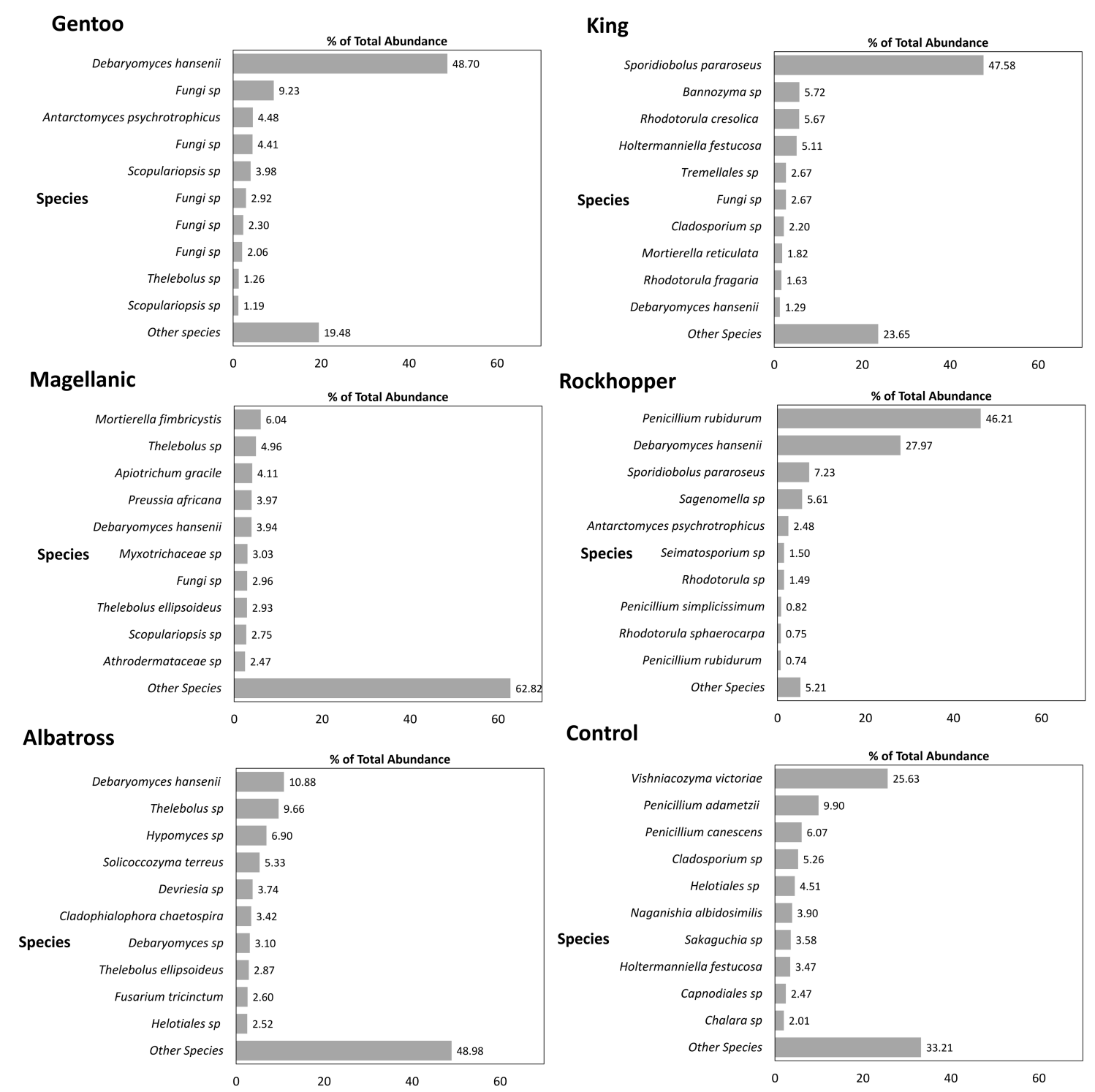

Figure 5 The ten most abundant OTUs in each site using abundance data from the 30,000 sequence subsampled OTU table. "Other Species" denotes the cumulative abundance of remaining species present from each site. The highest taxon level that was successfully assigned was used for each

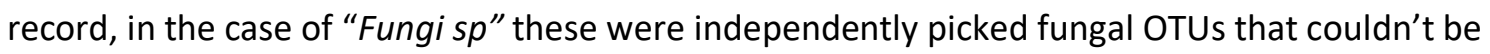
assigned any higher taxonomic rank. Due to clustering and OTU picking methodology, duplicate species can be recorded, as seen in Rockhopper (Penicillium rubidurum). 


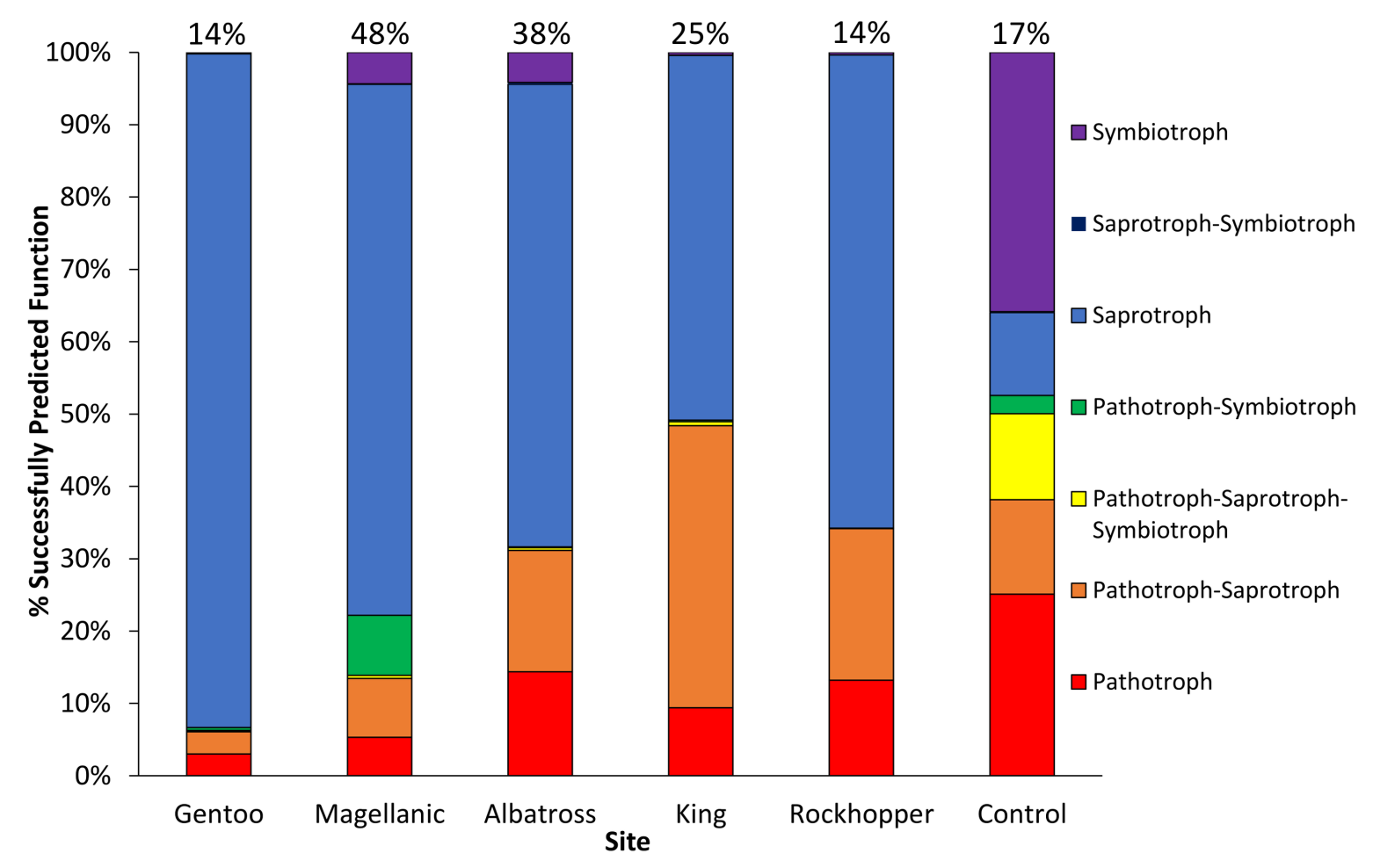

Figure 6 the trophic assignments of each site excluding the unassignable sequences, all successfully annotated OTUs were used to calculate the percentage abundance of each trophic strategy. The percentage of fungal sequences from each site successfully assigned to guilds by FUNGuild is shown above the bars. Only assignments of 'probable' and 'highly probable' were used. 